\title{
Dephasing in a Mach-Zehnder Interferometer by an Ohmic Contact
}

\author{
Edvin G. Idrisov, ${ }^{1,2}$ Ivan P. Levkivskyi, ${ }^{3,4,5}$ and Eugene V. Sukhorukov ${ }^{1}$ \\ ${ }^{1}$ Département de Physique Théorique, Université de Genève, CH-1211 Genève 4, Switzerland \\ ${ }^{2}$ Physics and Materials Science Research Unit, University of Luxembourg, L-1511 Luxembourg, Luxembourg \\ ${ }^{3}$ Theoretische Physik, ETH Zurich, CH-8093 Zurich, Switzerland \\ ${ }^{4}$ Institute of Ecology and Evolution, University of Bern, CH-3012 Bern, Switzerland \\ ${ }^{5}$ Department of Computational Biology, University of Lausanne, CH-1011 Lausanne, Switzerland
}

(Received 9 December 2017; published 9 July 2018)

\begin{abstract}
We study dephasing in an electronic Mach-Zehnder (MZ) interferometer based on quantum Hall edge states by a micrometer-sized Ohmic contact embedded in one of its arms. We find that at the filling factor $\nu=1$, as well as in the case where an Ohmic contact is connected to a MZ interferometer by a quantum point contact that transmits only one electron channel, the phase coherence may not be fully suppressed. Namely, if the voltage bias $\Delta \mu$ and the temperature $T$ are small compared to the charging energy of the Ohmic contact $E_{C}$, the free fermion picture is manifested, and the visibility saturates at its maximum value. At large biases, $\Delta \mu \gg E_{C}$, the visibility decays in a power-law manner.
\end{abstract}

DOI: 10.1103/PhysRevLett.121.026802

Recent progress in experimental techniques at nanoscale resulted in the emergence of a new field of "quantum electron optics," where, as the name suggests, electrons in onedimensional systems replace photons [1]. Typically, one uses for this purpose quantum Hall $(\mathrm{QH})$ edge states at integer filling factors, which play the role of the beams of photons, quantum point contacts (QPCs), that serve as beam splitters, and Ohmic contacts to inject equilibrium electrons. Despite many analogies with quantum optics, there is one important difference: quasi-one-dimensional electrons strongly interact, and often interactions cannot be considered perturbatively. Not only does this require an application of advanced theoretical methods, such as the bosonization technique [2], but it also leads to interesting physical effects related to electronic decoherence and relaxation which manifest themselves in Mach-Zehnder (MZ) interferometers [3,4], electronic relaxation measurements [5], HOM interferometry [6], and the heat Coulomb blockade (CB) effect [7].

Among various quantum electron optics devices, Ohmic contacts are the most intriguing systems, because in the context of the QH physics they present an example of strong coupling between completely different states of matter. This is especially the case at fractional fillings, where the strong theoretical effort has already been made [8], while experimentally the physics of Ohmic contacts is far from being fully understood. At integer filling factors, one often refers to earlier works of M. Büttiker, who proposed to consider Ohmic contacts as analogues of a black body in quantum optics, i.e., a reservoir of equilibrium electrons. According to the voltage probe [9] and dephasing probe [10] models, edge electrons entering an Ohmic contact are fully equilibrated, or lose completely their phase coherence. These models have been widely used in the literature [11].
Such an approach has some grounds in the case of chiral edge fermions at integer filling factors, where local correlation functions coincide with those for free fermions [12]. However, as has been pointed out in Ref. [13], in contrast to photons, electrons carry electrical charge, which has to be taken into account, if one considers an Ohmic contact from a somewhat broader perspective as a metallic island strongly coupled to edge states [14-16]. Indeed, even if the level spacing in such systems is negligible, the charging energy may compare to the base temperature of the experiment and other characteristic energies. As a result, such an Ohmic contact cannot fully equilibrate edge electrons [13]. A related phenomenon of the Coulomb blockade of the heat flux has recently been observed in the experiment [7].

In this Letter, we consider the dephasing probe model of an Ohmic contact and show that it fails in a particular case, where a metallic island with a finite charging energy is coupled to an arm of a MZ interferometer (see Fig. 1) via a QPC that transmits exactly one channel, as shown in Fig. 2 [17]. This is a rather strong statement, because it implies that electrons that enter and exit a metallic island are not statistically and quantum mechanically independent, despite the fact that the level spacing inside the island vanishes, and it can be considered a reservoir of neutral modes. This is because according to the effective theory of $\mathrm{QH}$ edge states [18] the phase difference of incoming and outgoing edge electrons is fully determined by the charge of the metallic island, and thus at energies lower than its charging energy there is no room for phase fluctuations. This statement is particularly interesting in the context of the existing hydrodynamic theory [19], suggesting that each edge electron carries infinite number of neutral modes. 


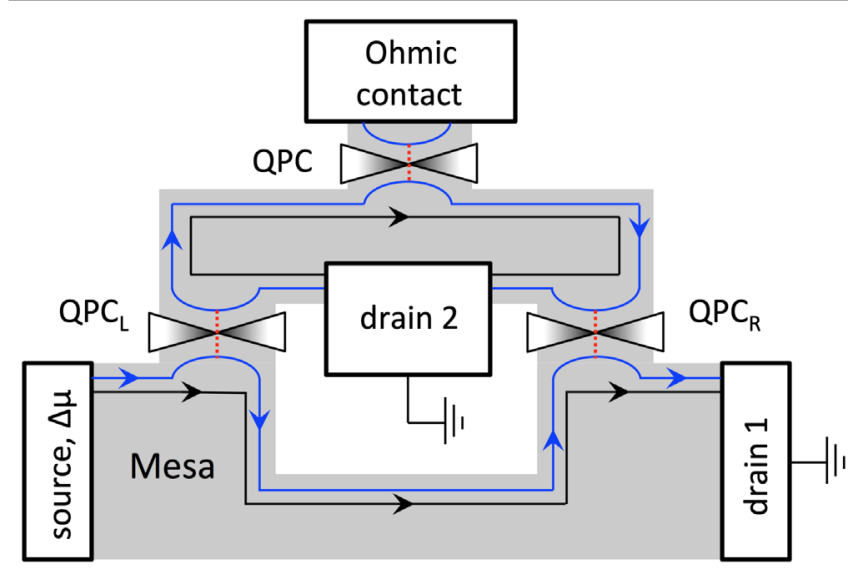

FIG. 1. Schematics of the electronic Mach-Zehnder interferometer based on a Corbino disk shaped $\mathrm{QH}$ system at filling factor $\nu=2$ (shown by the gray shaded area), so that two chiral edge states are formed (shown by arrowhead lines). The incoming outer edge state originates from the source, biased with the voltage $\Delta \mu$, and is split by $\mathrm{QPC}_{L}$ (quantum point contact) in two paths, which propagate in the upper and lower parts of the mesa and recombine at the $\mathrm{QPC}_{R}$. Thus, outer edge states enclose an $A B$ flux, which leads to $A B$ oscillations in the charge current, measured at the drain 2. An Ohmic contact is coupled to the edge state in the upper arm of the interferometer. It is a piece of metal, which absorbs incoming electron edge states and turns them into neutral electron-hole excitations. Then, it emits equilibrium neutral excitations as well as the charge current into the outgoing edge states. The number of channels transmitted to the Ohmic contact is controlled by the upper QPC. We assume, that at filling factor 2, the outer channel is fully transmitted to the Ohmic contact, while the inner channel can be both fully transmitted or fully reflected.

Such a scenario has been investigated with the help of the heat flux measurements with somewhat inconclusive results [5]. However, as we demonstrate in the Supplemental Material [20], adding collective modes to the edge leads to complete dephasing of an electron entering the Ohmic contact. Thus our proposal could also be considered as an ultimate test of the bosonic representation of the electron operator, which is the essential ingredient of the effective theory (absent in the hydrodynamic approach).

Model of Ohmic contact.-We consider an Ohmic contact as a piece of disordered metal of the finite geometrical capacitance $C$ strongly coupled to a $\mathrm{QH}$ edge [21]. We assume a capacitive interaction of electrons inside the Ohmic contact. The level spacing of neutral modes in it is negligible, while its charging energy $E_{C}=e^{2} / 2 C$ is finite. To take into account this fact, we follow the steps outlined in Ref. [13] and model neutral modes by elongating the electron channel inside the Ohmic contact to infinity, splitting it in two uncorrelated channels, and introducing the regularization parameter $\varepsilon$ in Eq. (3). A schematic representation of Ohmic contact is shown in Fig. 3. This minimal model agrees with the free-fermionic form of local correlation functions in the metallic island,

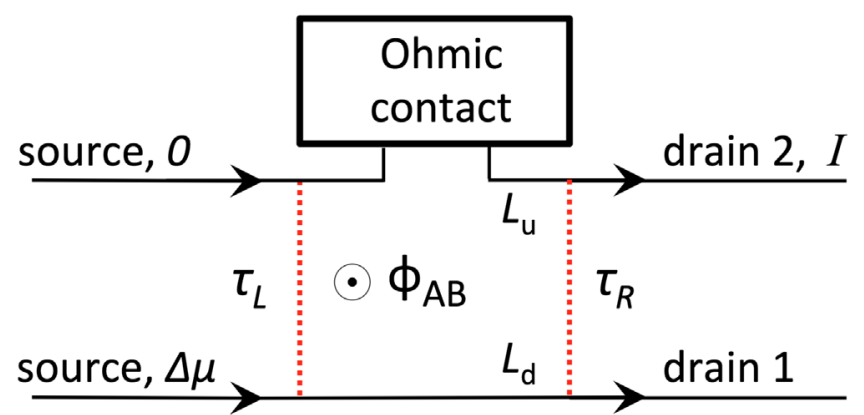

FIG. 2. The simplified scheme of the MZI is shown. It is obtained by cutting the interferometer in Fig. 1 at the drain 2, unfolding edge states, and leaving only outer edge state, since it contributes to the $A B$ oscillations. The tunneling amplitudes at the left and right QPCs are denoted by $\tau_{L}$ and $\tau_{R}$, respectively. $L_{u}$ and $L_{d}$ are the lengths of the upper and lower paths of interferometer. The voltage bias $\Delta \mu$ is applied to the lower channel. The current $I$ measured at the drain 2, and the differential conductance $G=d I / d \Delta \mu$ oscillate as a function of the $A B$ phase $\phi_{A B}$.

the absence of backscattering, the constant density of states at the Fermi level, and vanishing level spacing. A similar model has been successfully applied in the context of CB effect physics in Ref. [16] and agrees essentially perfectly with the experiment. Throughout the Letter, we set $e=\hbar=k_{B}=1$.

We use the low-energy effective theory to describe the QH edge states [18]. According to this theory, collective fluctuations of the charge densities in the electron channels $\rho_{\sigma}(x, t)=(1 / 2 \pi) \partial_{x} \phi_{\sigma}(x, t)$ are expressed in terms of the bosonic fields $\phi_{\sigma}(x, t)$, where the index $\sigma=-,+$ stands for incoming and outgoing channels, respectively. The bosonic fields satisfy the standard canonical commutation relation:

$$
\left[\partial_{x} \phi_{\sigma}(x, t), \phi_{\sigma^{\prime}}(y, t)\right]=2 \pi i \sigma \delta_{\sigma \sigma^{\prime}} \delta(x-y) .
$$

The Hamiltonian of the system consisting of edge states strongly coupled to the Ohmic contact includes two terms

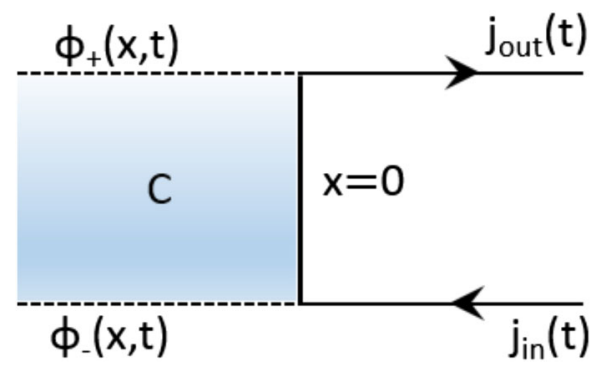

FIG. 3. An equivalent representation of the Ohmic contact at filling factor $\nu=1$. Edge states are described by two bosonic fields $\phi_{+}(x)$ and $\phi_{-}(x)$ of opposite chiralities. The region inside the Ohmic contact, where the capacitive interaction is assumed, is shown by the light blue color. 


$$
H=\frac{v_{F}}{4 \pi} \sum_{\sigma} \int_{-\infty}^{\infty} d x\left(\partial_{x} \phi_{\sigma}\right)^{2}+\frac{Q^{2}}{2 C}
$$

where

$$
Q=\int_{-\infty}^{0} d x e^{\varepsilon x / v_{F}}\left[\rho_{+}(x)+\rho_{-}(x)\right]
$$

is an operator of the total charge accumulated at the Ohmic contact, and $\varepsilon$ is the regularization parameter. The first term in Eq. (2) is the kinetic energy part of the Hamiltonian, which describes the dynamics of incoming and outgoing edge channels. The second term is the charging energy of the Ohmic contact of a finite size.

Using commutation relations (1) and the Hamiltonian (2), we write the equations of motion for the bosonic fields $\phi_{\sigma}(x, t)$ :

$$
\sigma \partial_{t} \phi_{\sigma}(x, t)+v_{F} \partial_{x} \phi_{\sigma}(x, t)=-\frac{Q(t) e^{\varepsilon x / v_{F}}}{C} \theta(-x) .
$$

These equations have to be complemented with the following boundary conditions:

$$
\begin{aligned}
\partial_{t} \phi_{+}(-\infty, t) & =-2 \pi j_{s}(t), \\
\partial_{t} \phi_{-}(0, t) & =2 \pi j_{\text {in }}(t),
\end{aligned}
$$

where $j_{\text {in }}(t)$ is the current flowing into the Ohmic contact, while $j_{s}(t)$ (source current) describes equilibrium fluctuations of the neutral mode with the temperature $T$. Solving equations (4) with the boundary conditions (5), one relates the outgoing current $j_{\text {out }}(t)=-\partial_{t} \phi_{+}(0, t) / 2 \pi$ to the incoming current $j_{\text {in }}(t)$, as shown in Fig. 2:

$$
j_{\text {out }}(\omega)=\frac{i \omega R_{q} C}{i \omega R_{q} C-1} j_{s}(\omega)-\frac{1}{i \omega R_{q} C-1} j_{\text {in }}(\omega),
$$

where $R_{q}=2 \pi \hbar / e^{2}$ is a quantum of resistance (restoring natural unites).

The statistics of current fluctuations $\delta j_{\alpha}(\omega) \equiv$ $j_{\alpha}(\omega)-\left\langle j_{\alpha}(\omega)\right\rangle$, where $\alpha=$ in, $s$, is characterized by the equilibrium density function $S(\omega)$ [22]

$$
S(\omega)=\frac{\omega / R_{q}}{1-e^{-\omega / T}},
$$

defined via the relation

$$
\left\langle\delta j_{\alpha}(\omega) \delta j_{\beta}\left(\omega^{\prime}\right)\right\rangle=2 \pi \delta_{\alpha \beta} \delta\left(\omega+\omega^{\prime}\right) S(\omega) .
$$

Equations (5)-(8) can now be used to calculate two-point correlation functions of the fields $\phi_{\sigma}(x, t)$.

Electronic MZ interferometer.-This type of electronic interferometers utilizes a two-dimensional system in the form of so-called Corbino disk and two QPCs mixing edge channels so as to avoid backscattering [3]. Schematic representation of an electronic MZI with an Ohmic contact attached to it is shown in Figs. 1, 2. Two point contacts located at positions $x_{l}$, where $l=L, R$ (left, right), mix the edge states and allow interference between them. This can be described by the tunneling Hamiltonian with the vertex operators at $x_{l}[12]$

$$
\begin{aligned}
H_{T} & =A_{L}+A_{R}+\text { H.c. }, \\
A_{l} & \propto \tau_{l} \exp \left[-i \phi_{u}\left(x_{l}\right)+i \phi_{d}\left(x_{l}\right)\right],
\end{aligned}
$$

where $\tau_{l}$ are the tunneling coupling amplitudes, and $\phi_{i}\left(x_{l}\right), i=u, d$, are the bosonic fields at the upper and lower channel of the MZ interferometer, respectively. The $A B$ phase is included in the tunneling amplitudes via the relation $\tau_{R}^{*} \tau_{L}=\left|\tau_{R}\right|\left|\tau_{L}\right| e^{i \phi_{A B}}$. We investigate interference effects in the electron tunneling current. The total tunneling current consists of three terms, namely, $I=I_{L L}+I_{R R}+2 \operatorname{Re}\left(I_{R L}\right)$, where the third term contains the $A B$ phase [20]. The degree of the phase coherence is characterized by the visibility of $A B$ oscillations

$$
V=\frac{G_{\max }-G_{\min }}{G_{\max }+G_{\min }},
$$

where $G=\partial_{\Delta \mu} I$ is the differential conductance associated with tunneling current $I$. In the rest of the Letter we investigate the dependence of the visibility on the temperature $T$ and applied bias $\Delta \mu$. The details of the calculations are presented in the Supplemental Material [20]. Here, we mention only that in order to evaluate the average current $I$ (as well differential conductance $G$ ), we use Eqs. (5)-(9) and the Gaussian character of the theory.

Direct current and conductance.-In our model the interaction is present only in the Ohmic contact located between points $x_{L}$ and $x_{R}$ [17]. The important consequence of this fact is that the interaction cannot affect the direct contribution $I_{\mathrm{dir}}=I_{L L}+I_{R R}$, which also follows from the unitarity relation of scattering (6). Therefore, we readily obtain the direct part of total current

$$
I_{\mathrm{dir}}=\frac{\left|\tau_{L}\right|^{2}+\left|\tau_{R}\right|^{2}}{2 \pi v_{F}^{2}} \Delta \mu .
$$

Thus, the direct conductance $G_{\mathrm{dir}}=\partial_{\Delta \mu} I_{\mathrm{dir}}$,

$$
G_{\mathrm{dir}}=\frac{\left|\tau_{L}\right|^{2}+\left|\tau_{R}\right|^{2}}{2 \pi v_{F}^{2}}
$$

shows conventional Ohmic behavior; i.e., it is independent of the temperature $T$ and bias $\Delta \mu$.

Visibility of $A B$ oscillations. Low-bias Ohmic regime.We consider the oscillating part of the conductance, $G_{\mathrm{osc}} \equiv$ $\partial_{\Delta \mu}\left[2 \operatorname{Re}\left(I_{\mathrm{RL}}\right)\right]$, focus on the regime of low bias, and take 
the limit $\Delta \mu \rightarrow 0$. At low temperatures, $T \ll E_{C}$, the behavior is the same as for noninteracting fermions [20]:

$$
G_{\mathrm{osc}}=\frac{\left|\tau_{L}\right|\left|\tau_{R}\right|}{\pi v_{F}^{2}} \cos \left(\phi_{A B}\right)
$$

and according to the Eq. (10) the visibility acquires the following form:

$$
V \equiv V_{0}=\frac{2\left|\tau_{L}\right|\left|\tau_{R}\right|}{\left|\tau_{L}\right|^{2}+\left|\tau_{R}\right|^{2}}
$$

Thus, at low temperatures, $T \ll E_{C}$, thermal fluctuations are not able to suppress the quantum coherence of edge states despite the fact that they are strongly coupled to an Ohmic contact.

In the opposite limit of high temperatures, $T \gg E_{C}$, we obtain the following result for the oscillating part of the conductance [20]

$$
G_{\mathrm{osc}}=\frac{\left|\tau_{L}\right|\left|\tau_{R}\right|}{v_{F}^{2}} \sqrt{\frac{\pi T}{E_{C}}} e^{-\pi^{2} T / E_{C}} \cos \left(\phi_{A B}\right) .
$$

Next, substituting Eqs. (12) and (15) into the Eq. (10), we obtain

$$
V / V_{0}=\pi \sqrt{\frac{\pi T}{E_{C}}} e^{-\pi^{2} T / E_{C}} .
$$

The dependence of $V / V_{0}$ on temperature is given in Fig. 4.

Visibility of $A B$ oscillations. Nonlinear regime.-In this section we focus on the nonlinear regime, namely, $\Delta \mu$ is arbitrary, and $T \rightarrow 0$. In the case of small bias, $\Delta \mu \ll E_{C}$,

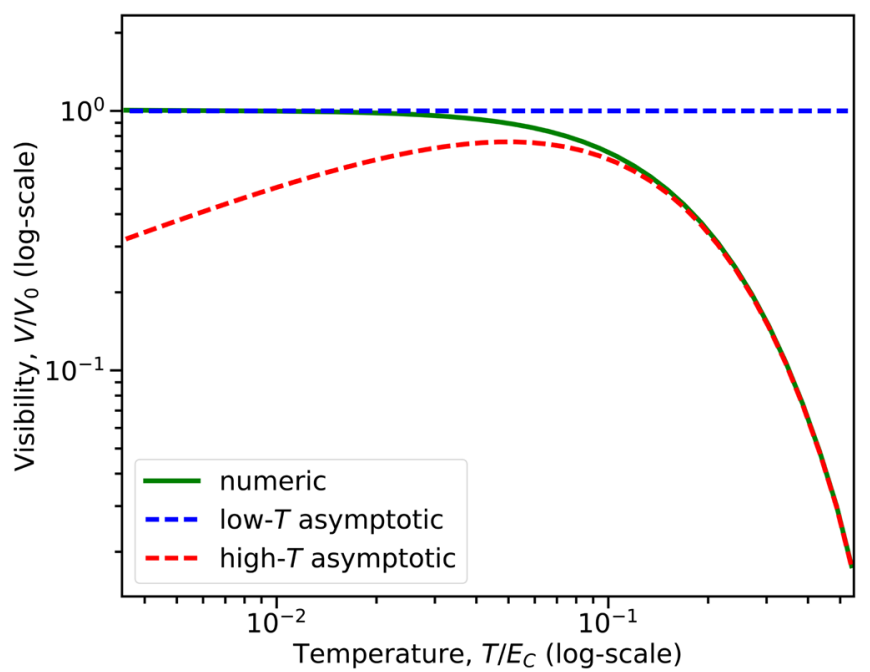

FIG. 4. The normalized visibility $V / V_{0}$ is plotted versus the dimensionless temperature $T / E_{C}$ in $\log -\log$ scale (here $T \neq 0$, $\Delta \mu \rightarrow 0)$. the free-fermionic behavior is restored [20], and the visibility is given by Eq. (14). In the case of the large bias, $\Delta \mu \gg E_{C}$, we obtain the following result [20] for the oscillating (coherent) part of the current, $I_{\text {osc }}=2 \operatorname{Re}\left(I_{R L}\right)$, including the subleading term in the bias:

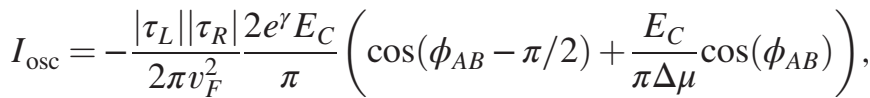

where $\gamma \approx 0.5772$ is an Euler constant. Interestingly, at biases larger than the charging energy $E_{C}$ of the Ohmic contact the coherent contribution to the current saturates at values $\propto E_{C}$. This implies that it possibly originates from the elastic tunneling induced by the resonance in the transmission of plasmons in the upper arm of the interferometer. Combining Eqs. (10), (11), and (17), one arrives at the following expression for the visibility of $A B$ oscillations

$$
V / V_{0}=\frac{e^{\gamma} E_{C}^{2}}{(\pi \Delta \mu)^{2}} .
$$

The full dependence of $V / V_{0}$ on the bias is shown in Fig. 5 .

Finally, we note that at filling factor $\nu \geq 2$, or more generally, when an Ohmic contact is perfectly coupled to at least two electron channels, the phase coherence is fully suppressed, as shown in the Supplemental Material [20]. This can easily be explained by the fact that it does not cost energy to flip the pseudospin related to extra electron channels, because the level spacing of neutral modes in the Ohmic contact is assumed to be zero.

To summarize, we have studied the dephasing mechanism in the electronic MZ interferometer based on the edge states in a $\mathrm{QH}$ system at filling factor $\nu=1$, strongly

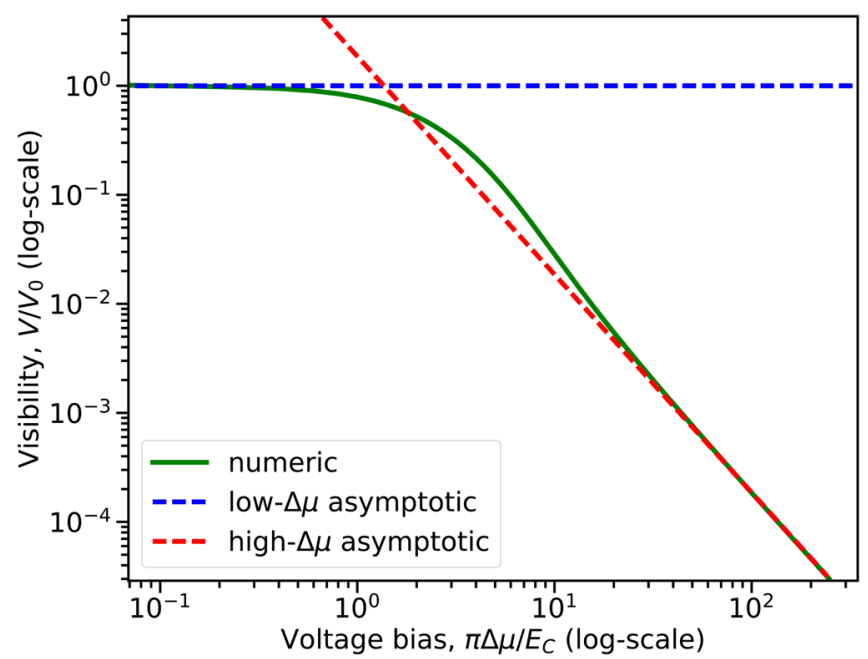

FIG. 5. The normalized visibility $V / V_{0}$ is plotted versus the dimensionless bias $\pi \Delta \mu / E_{C}$ in $\log -\log$ scale (here $T \rightarrow 0$, $\Delta \mu \neq 0)$. 
coupled to an Ohmic contact. Alternatively, an Ohmic contact may be connected to an interferometer by a QPC, transmitting only one electron channel. We have used a simple model of an Ohmic contact as a reservoir of neutral modes with the finite charging energy $E_{C}$. It was shown earlier [13] that such an Ohmic contact is not always able to fully equilibrate edge states. Here, we have demonstrated that it is also not always able to fully suppress the phase coherence of edge electrons. This is because edge electrons carry charge, and at filling factor $\nu=1$ the phase of an electron is determined by the charge accumulated at an Ohmic contact. At temperatures and voltage biases smaller than the charging energy $E_{C}$, charge fluctuations, and consequently phase fluctuations are suppressed. On the other hand, at filling factors larger than 1 additional degrees of freedom of the edge electrons are perfectly coupled to neutral modes in the Ohmic contact, which leads to the full suppression of the phase coherence [20].

We acknowledge support from the Swiss NSF. E. I. acknowledges financial support from the Fonds National de la Recherche Luxembourg under Grant No. ATTRACT 7556175 .

[1] For a review, see E. Bocquillon, V. Freulon, F. D. Parmentier, J.-M. Berroir, B. Plaçais, C. Wahl, J. Rech, T. Jonckheere, T. Martin, C. Grenier, D. Ferraro, P. Degiovanni, and G. Fève, Ann. Phys. (Amsterdam) 526, 1 (2014); B. Roussel, C. Cabart, G. Fève, E. Thibierge, and P. Degiovanni, Phys. Status Solidi B 254, 1600621 (2017); A. Marguerite, E. Bocquillon, J.-M. Berroir, B. Plaçais, A. Cavanna, Y. Jin, P. Degiovanni, and G. Fève, Phys. Status Solidi B 254, 1600618 (2017).

[2] Th. Giamarchi, Quantum Physics in One Dimension (Oxford University Press, Oxford, 2003).

[3] E. Bieri, M. Weiss, O. Göktas, M. Hauser, C. Schönenberger, and S. Oberholzer, Phys. Rev. B 79, 245324 (2009); L. V. Litvin, H.-P. Tranitz, W. Wegscheider, and C. Strunk, Phys. Rev. B 75, 033315 (2007); I. Neder, F. Marquardt, M. Heiblum, D. Mahalu, and V. Umansky, Nat. Phys. 3, 534 (2007); P. Roulleau, F. Portier, D. C. Glattli, P. Roche, A. Cavanna, G. Faini, U. Gennser, and D. Mailly, Phys. Rev. B 76, 161309 (2007).

[4] S. Tewari, P. Roulleau, C. Grenier, F. Portier, A. Cavanna, U. Gennser, D. Mailly, and P. Roche, Phys. Rev. B 93, 035420 (2016); A. O. Slobodeniuk, E. G. Idrisov, and E. V. Sukhorukov, Phys. Rev. B 93, 035421 (2016); A. S. Goremykina and E. V. Sukhorukov, Phys. Rev. B 97, 115418 (2018).

[5] H. le Sueur, C. Altimiras, U. Gennser, A. Cavanna, D. Mailly, and F. Pierre, Phys. Rev. Lett. 105, 056803 (2010).

[6] A. Marguerite, C. Cabart, C. Wahl, B. Roussel, V. Freulon, D. Ferraro, Ch. Grenier, J.-M. Berroir, B. Plaçais, T.
Jonckheere, J. Rech, T. Martin, P. Degiovanni, A. Cavanna, Y. Jin, and G. Fève, Phys. Rev. B 94, 115311 (2016).

[7] E. Sivre, A. Anthore, F. D. Parmentier, A. Cavanna, U. Gennser, A. Ouerghi, Y. Jin, and F. Pierre, Nat. Phys. 14, 145 (2018).

[8] C. L. Kane and M. P. A. Fisher, Phys. Rev. B 52, 17393 (1995); C. C. Chamon and E. Fradkin, Phys. Rev. B 56, 2012 (1997); V. V. Ponomarenko and D. V. Averin, Phys. Rev. B 70, 195316 (2004).

[9] M. Büttiker, IBM J. Res. Dev. 32, 63 (1988).

[10] M. J. M. de Jong and C. W. J. Beenakker, Physica (Amsterdam) 230A, 219 (1996).

[11] For a review, see Y. M. Blanter and M. Büttiker, Phys. Rep. 336, 1 (2000).

[12] I. P. Levkivskyi and E. V. Sukhorukov, Phys. Rev. B 78, 045322 (2008).

[13] A. O. Slobodeniuk, I. P. Levkivskyi, and E. V. Sukhorukov, Phys. Rev. B 88, 165307 (2013).

[14] S. Jezouin, M. Albert, F. D. Parmentier, A. Anthore, U. Gennser, A. Cavanna, I. Safi, and F. Pierre, Nat. Commun. 4, 1802 (2013); S. Jezouin, F. D. Parmentier, A. Anthore, U. Gennser, A. Cavanna, Y. Jin, and F. Pierre, Science 342, 601 (2013).

[15] Z. Iftikhar, S. Jezouin, A. Anthore, U. Gennser, F. D. Parmentier, A. Cavanna, and F. Pierre, Nature (London) 526, 233 (2015); Z. Iftikhar, A. Anthore, A. K. Mitchell, F. D. Parmentier, U. Gennser, A. Ouerghi, A. Cavanna, C. Mora, P. Simon, and F. Pierre, arXiv:1708.02542.

[16] A. Furusaki and K. A. Matveev, Phys. Rev. B 52, 16676 (1995); S. Jezouin, Z. Iftikhar, A. Anthore, F. D. Parmentier, U. Gennser, A. Cavanna, A. Ouerghi, I. P. Levkivskyi, E. Idrisov, E. V. Sukhorukov, L. I. Glazman, and F. Pierre, Nature (London) 536, 58 (2016); E. G. Idrisov, I. P. Levkivskyi, and E. V. Sukhorukov, Phys. Rev. B 96, 155408 (2017).

[17] The length of arms, $L_{i}$, where $i=u, d$, is assumed to be small, namely, $\Delta \mu L_{i} / v_{F} \ll 1$ and $T L_{i} / v_{F} \ll 1$, where $T$ is the bath temperature. This assumption allows us to neglect the interaction of edge electrons, because it only weakly contributes to dephasing and to the capacitance of the metallic island.

[18] X. G. Wen, Phys. Rev. B 41, 12838 (1990).

[19] I. L. Aleiner and L. I. Glazman, Phys. Rev. Lett. 72, 2935 (1994).

[20] See Supplemental Material at http://link.aps.org/ supplemental/10.1103/PhysRevLett.121.026802 for the detailed calculations of conductance and visibility.

[21] Because of strong coupling of the Ohmic contact to the edge state there is no sign of conventional Coulomb blockade phenomenon in the present model; namely, there is no charge quantization nor any Coulomb induced reduction of the electrical conductance. However, there is a heat Coulomb blockade of one ballistic channel. See Ref. [7].

[22] E. M. Lifshitz and L.P. Pitaevskii, Statistical Physics, Part 2: Landau and Lifshits Course of Theoretical Physics (Butterworth-Heinemann, Oxford, 1980), Vol. 9. 